\title{
1 Limits to environmental masking of genetic quality in sexual signals
}

3 James Malcolm Howie ${ }^{\mathrm{a}, \mathrm{b}}$, james.howie.11@ucl.ac.uk

4 Harry Alexander Cordeaux Dawson ${ }^{a}$, hacdawson98@gmail.com

$5 \quad$ Andrew Pomiankowski ${ }^{\text {a, c }}$, ucbhpom@ucl.ac.uk

6 Kevin Fowler ${ }^{\text {a }}$, k.fowler@ucl.ac.uk

7

8 a Department of Genetics, Evolution and Environment, University College London, Gower Street,

9 London, WC1E 6BT, UK;

$10{ }^{\mathrm{b}}$ Institute of Population Genetics, University of Veterinary Medicine, Veterinäplatz 1, 1210

11 Vienna, Austria

$12{ }^{\mathrm{c}}$ CoMPLEX, University College London, Gower Street, London, WC1E 6BT, UK

14 Subject: Evolution

15 Keywords: condition dependence, GxE, indirect genetic benefits, sexual ornament,

16 sexual selection, stalk-eyed fly

17 Author for correspondence: Andrew Pomiankowski, ucbhpom@ucl.ac.uk

18 Orcid: 0000-0002-5171-8755

19 Running Head: GxE sexual signals 


\section{Abstract}

23 There is considerable debate over the value of male sexual ornaments as signals of

24 genetic quality. Studies alternately report that environmental variation enhances or

25 diminishes the genetic signal, or leads to crossover where genotypes perform well in one

26 environment but poorly in another. A unified understanding is lacking. We conduct the

27 first experimental test examining the dual effects of distinct low and high genetic quality

28 (inbred versus crossed parental lines) and low, through high, to extreme environmental

29 stress (larval diets) on a condition-dependent male ornament. We find that differences in

30 genetic quality signalled by the ornament (male eyespan in Diasemopsis meigenii stalk-

31 eyed flies) become visible and are amplified under high stress but are overwhelmed in

32 extreme stress environments. Variance among distinct genetic lines increases with

33 environmental stress in both genetic quality classes, but at a slower rate in high quality

34 outcrossed flies. Individual genetic lines generally maintain their ranks across

35 environments, except among high quality lines under low stress conditions, where low

36 genetic variance precludes differentiation between ranks. Our results provide a

37 conceptual advance, demonstrating a unified pattern for how genetic and

38 environmental quality interact. They show when environmental conditions lead to the

39 amplification of differences in signals of genetic quality and thereby enhance the

40 potential indirect genetic benefits gained by female mate choice. 


\section{1. Introduction}

43

44 Many exaggerated male sexual ornaments are thought to have evolved to provide

information about the genetic quality of the signaller $[1,2,3,4,5,6,7]$. Yet these traits typically also respond strongly to environmental variation $[8,9,10,11]$, and it is unclear what impact this has on their signalling function $[12,13]$. Does increasing environmental stress expose the underlying genetic differences in quality or mask them by overwhelming the genetic signal? Different studies have variously reported that environmental variation enhances $[1,2,14,15]$ or diminishes $[16,17,18]$ the phenotypic signal of genetic quality. Others reveal crossover, where genotypes that perform well in one environment do poorly in another $[17,19,20,21]$. These contrasting outcomes arise from a lack of consistency in experimental approach. The main problem is that analysis has focussed on genetic variation rather than distinct classes of genetic quality, coupled to a limited rather than wide range of environmental stress. We present a novel experimental design that addresses both of these deficits, which leads us to propose a unified understanding of how variation in genetic quality is impacted by environmental variation. This gives a far clearer understanding of the conditions under which sexual display traits can function to accurately reveal the genetic quality of signallers [22].

In this study, we adopt an integrated experimental approach, and for the first time examine the impact of a similar wide range in both genetic and environmental quality. We chose to focus specifically on male eyespan variation in the stalk-eyed fly $[23,24]$ as this trait has been subject to extensive previous work. Male eyespan is highly exaggerated due to female choice $[11,25,26,27,28,29,30,31,32]$ and also functions as a signal in male-male antagonistic interactions over mate mating sites [25,33,34]. It is 
67 highly condition-dependent relative to other traits in relation to both genetic [24, but see $36]$ and environmental $[9,11,35]$ stressors, and is responsive to a range of environmental stress types $[9,11,24,35,37,38]$, while genetically distinct families have also been shown to respond differently to environmental stress [1].

72 Our novel experimental design to study genetic quality-by-environment ( $\mathrm{G} \times \mathrm{E})$ interactions in signalling traits exploits pre-defined genetic $[39,40,41]$ and environmental quality classes. To vary genetic quality, crosses were made within or between a set of parental inbred lines $(f \sim 0.908$ [42]; Figure 1). This allowed us to compare low genetic quality, highly homozygous "incross" lines $(n=16$, crosses $=67)$ with high genetic quality, highly heterozygous "outcross" lines $(n=17$, crosses $=50)$. We used incross and outcross lines not to study the effect of inbreeding per se, but because previous work unambiguously shows they correspond to low and high genetic quality classes

80 respectively [24]. The large number of independent crosses within or between lines

81 allows us to capture the contribution of genetic variation in the sexual ornament among

82 low and high genetic quality classes.

84 We likewise generated a wide range of environmental quality variation through reductions in the amount of food available to developing larvae. This approach is a wellestablished method for creating stress in holometabolous insects

$87[1,11,15,26,30,35,37,40,43,44,45]$ and has been used extensively in prior stalk-eyed fly

88 studies $[9,11,24,30]$, where it generates body size variation equivalent to the range found 89 in natural populations [32,35]. Eggs were collected from each cross, and reared under

90 conditions of low, high and extreme environmental stress. Intermediate levels of stress 


\section{$94 \quad$ \# Figure 1.}

95

96

97

98

99

100

101

102

103

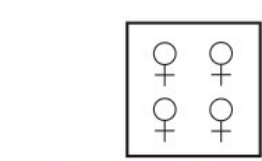

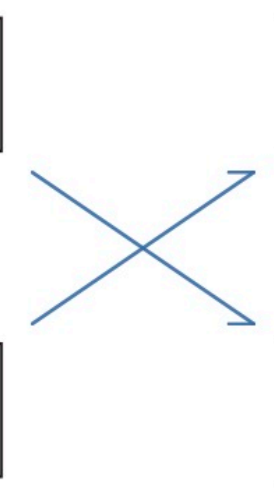

Line 1
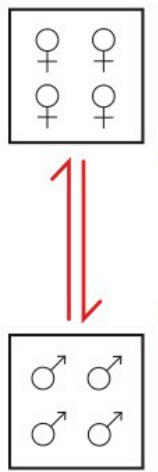

Line 2

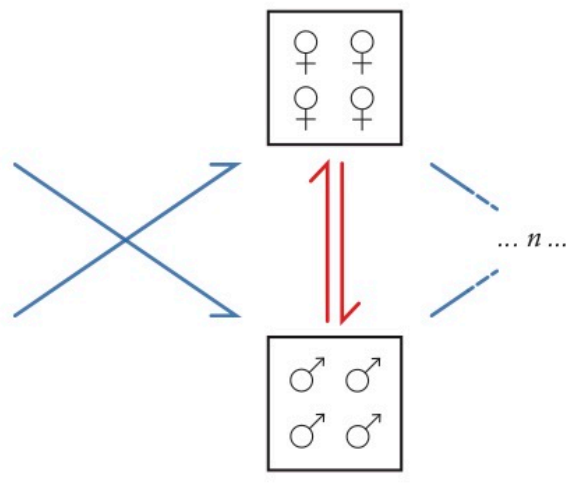

Line 3

104

105 Fig. 1. The crossing protocol used to generate incross and outcross offspring. Each

106 inbred line was crossed with itself to create incross flies (red), or with another inbred line

107 to create outcross flies (blue). Each cross used 4 males and 4 females and was repeated to

108 generate two families per cross. \#

109

110

111

112

113

114

115

116 
117 flies from 117 experimental crosses), and because those stress levels have been

118 extensively investigated previously [11]. The extreme level was defined as the least food

119 level where larval viability was not seriously impaired (see below)

121 Our use of the terminology low/high genetic quality and low/high/extreme

122 environmental quality is necessarily arbitrary but justified in terms of the experimental

123 design and in the results that follow. We comment further on these definitions in the

124 Discussion. The innovation in our experimental design lies in delivering controlled

125 manipulation of genetic quality and environmental stress, over several pre-defined

126 quality levels, thereby enabling an in depth exploration of genetic quality-by-

127 environment $(\mathrm{G} \times \mathrm{E})$ variation in a male sexual ornament. We use this to investigate the

128 signalling utility of the male ornament in providing information about indirect genetic

129 benefits through female mate choice.

\section{$131 \quad 2$. Material and methods}

\section{3 (a) Production of experimental flies}

134 A suite of 105 inbred lines were founded from a stock of Diasemopsis meigenii [24], an

135 African stalk-eyed fly, derived from flies collected in South Africa by S. Hilger in 2000.

136 After 11 generations of full-sib mating, the extant lines were bred in cage culture ( 200

137 flies/cage). For this study, 17 lines that varied between generations $F_{24}-F_{33}$ were chosen

138 as parental lines to use in crosses, with flies collected as eggs laid on petri dishes

139 containing excess puréed sweet corn $(10 \mathrm{~g})$. At eclosion, flies were placed in large cages

140 (151), separated by sex ( $\sim 2$ weeks) and raised until sexual maturity ( $\sim 10$ weeks). Adults

141 were fed puréed sweet corn with antifungal Nipagin, replenished twice per week. All 
142 flies were maintained using our standard protocol at $25^{\circ} \mathrm{C}$ on a $12: 12$ hour light:dark

143 cycle, with fifteen-minute artificial "dawn" and "dusk" periods (reduced illumination) at

144 the start and end of the light phase throughout the experiment.

\section{6 (b) Variation in genetic and environmental effects}

147 Variation in genetic quality was achieved using previously created inbred lines [24] in a

148 crossing protocol (Figure 1; modified from [36] and [24]). "Incross" flies were created

149 from male-female crosses within an inbred line and "outcross" flies were created from

150 crosses between different inbred lines. In each cross, 4 adult males from line $x$ and 4

151 adult females from line $y$ were allowed to mate in a 1.51 pot $(x=y$ for incross, $x \neq y$ for

152 outcross). Reciprocal male $x$ - female $y$ and female $x$-male $y$ pots were set up. Multiple

153 replicates of each cross (between 1-8) were set up, with higher rates for inbred crosses as

154 they were less fecund. Eggs were collected twice weekly over 23 days. In all, 142 crosses

155 were set up, of which 117 generated sufficient offspring across the food treatments: 67

156 incrosses of 15 inbred lines and 50 outcrosses between 16 pairs of inbred lines. An inbred

157 line was used in an incross the same number of times as it was used in an outcross, and

158 as far as possible equal numbers of live adult males and females were collected from each

159 line, to balance sex chromosomal, cytoplasmic and other male/female parental effects.

161 Incross flies have low genetic quality as they are highly homozygous, being derived from

162 inbred lines created by repeated brother-sister pairings (11 generations), with an expected

163 inbreeding coefficient of $f \sim 0.908$ [24]. In contrast, outcross flies have high genetic

164 quality as they are expected to be heterozygous for most of the alleles fixed in the

165 parental inbred lines from which they are derived. Although the terms - low and high

166 genetic quality - are arbitrary, there was evidence of substantial heterosis in a variety of 
167 traits when inbred flies were crossed, so the terms reflect the nature of these two genetic

168 groups [24].

169

170 For each incross or outcross, fertilised eggs were placed in groups of 5 in petri dishes

171 containing two cotton pads, $15 \mathrm{ml}$ water and $5 \mathrm{ml}$ of food medium. Three qualities of

172 food medium were used with "pure" corn diluted with water at ratios of 1:1, 1:10, and

173 1:20, which we designate as "low", "high" and "extreme" stress respectively. "Pure"

174 corn was made by forcing puréed sweet corn kernels through a fine sieve to remove

175 husks and provide homogeneity. Food qualities were chosen based on a pilot study, with

176 levels of food stress used that were found to lie within normal rates of egg-adult survival

177 (Figure 2, see SI.C). Although the terms - low, high and extreme environmental stress -

178 are again arbitrary, they nonetheless capture particular qualities. The low food level was

179 similar to the standard media on which larvae are raised. The high food level was used

180 previously where it was associated with reduced size in a variety of traits [24]. The

181 extreme food level constitutes the far end of the stress spectrum before differential

182 survival is evident (Figure 2). In the range used (i.e. 1:1 to 1:20), egg-to-adult survival did

183 not differ in the pilot, and was at $\sim 50 \%$. We did not go beyond this level, as a serious

184 loss of adults would have placed greater logistical difficulties in delivering the already

185 considerable sample size in the experiment. In the main experiment, a census of pupae

186 was additionally made as a measure of survival for each cross in each environment.

188 (c) Adult morphology

189 After eclosion, flies of each cross were collected and frozen at $-20^{\circ} \mathrm{C}$. All males were

190 measured for eyespan (the distance between the outermost tips of the eyes $[9,46]$ ) and

191 thorax (the distance between the centre of the most posterior point of the head to the joint 


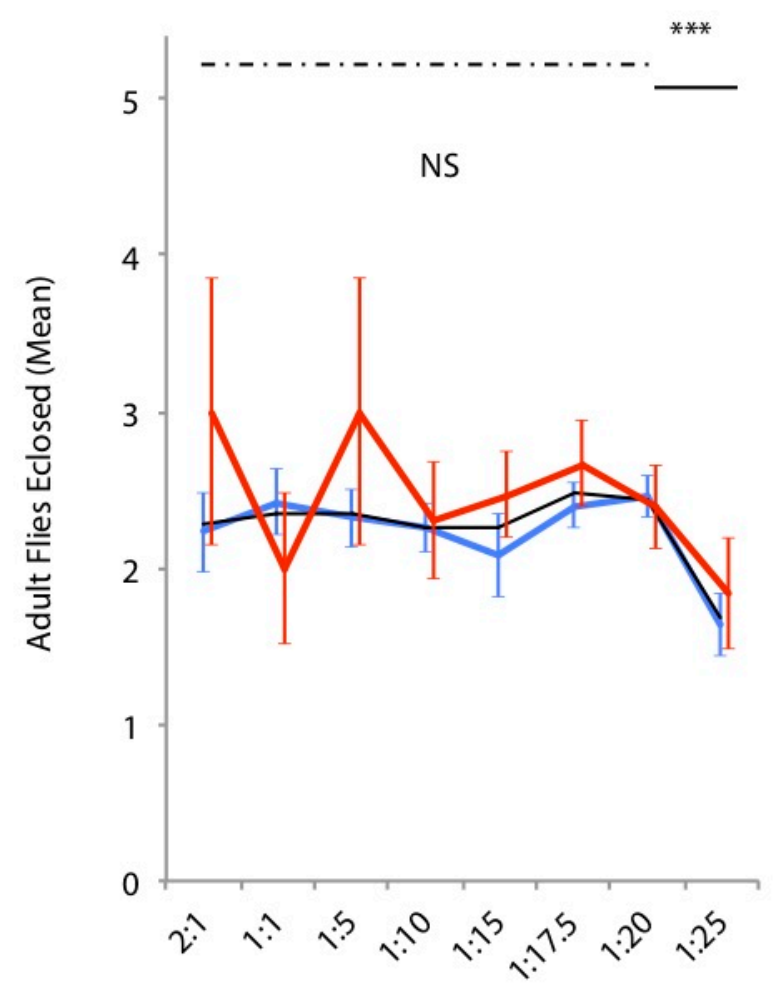

Food Stress Level

207 Fig. 2. Mean number of adult flies eclosing per petri dish ( \pm SE) given seeding with five

208 eggs, when subject to different larval treatments (ratio of corn:water), for inbred lines

209 (red) and stock (blue), or when pooled (black). Pairwise comparison of adjacent

210 treatments showed a significant drop in survival between the adjacent 1:20 and 1:25

211 treatments (solid line, ${ }^{* * *} P<0.001$ ), and no difference between other adjacent levels

212 (dashed line, NS). A similar pattern was observed for inbred and stock considered

213 separately across the adjacent 1:20 and 1:25 treatments (both $P<0.001)$. Inbred and

214 stock populations did not differ at any food level (all $P>0.05$ ). Data is based on a pilot

215 experiment (17 crosses, 10 stock, 7 inbred, $N=218$ stock, 68 inbred; details SI.C). \# 
217 between the meta-thoracic legs and the thorax $[47,48]$ ) to a tolerance of $0.01 \mathrm{~mm}$, using a

218 video camera mounted on a monocular microscope and ImageJ image capture software

219 v.1.46 [49]. The repeatability of these morphological trait measurements is very high at

$220>99 \%$ [9]. In total 1186 males were phenotyped. All measurements were made blind by

$221 \mathrm{JMH}$. In a few cases $(n=9)$, a measurement was not included in the dataset due to sample

222 damage.

223

\section{4 (d) Statistical analysis}

225 To test for effects of incross/outcross genetic quality (G), environmental (E) and the $\mathrm{G} x$

226 E interaction on morphological trait variation, several general linear mixed effects

227 models (GLMMs) were fitted via REML. In each model, G, E and their interaction were

228 included as fixed effects. Male parental line and female parental line were included as

229 random effects, as was cross and its interaction with E. Additional random effects of

230 male line $x \mathrm{E}$, male line $\mathrm{x} \mathrm{G}$, female line $\mathrm{x} \mathrm{E}$ and female line $\mathrm{x} \mathrm{G}$ explained zero variance

231 and so were removed in model simplification. GLMMs for male eyespan had thorax

232 added as a covariate to control for body size. Thorax length accounted for a significant

233 portion of variance, but its addition did not substantially alter the results (for

234 completeness, analyses of absolute trait values are given in the SI.A). GLMM models

235 fitted pairwise to low versus high and high versus extreme environmental stress were

236 used to further investigate the basis of the observed G x E patterns, as finally were two-

237 tailed $t$-tests at each level of $\mathrm{E}$ to test whether incross male eyespan was larger or smaller

238 than outcross male eyespan.

240 Coefficients of variation (CVs), the ratio of the standard deviation to the mean, were

241 used to assess how variance in male eyespan responded to genetic quality, 
242 environmental and $\mathrm{G} \times \mathrm{E}$ stress. CVs control for changes in variance purely as a function

243 of size, and are considered to be less biased than heritability estimates in $\mathrm{G} \times \mathrm{E}$ studies

244 [50]. Least square means for male relative eyespan were extracted from GLMMs for

245 each cross, for each E and G, to calculate among-cross CVs. Among-cross CVs were

246 then compared between incross and outcross using modified signed-likelihood ratio tests

247 (M-SLRT; [51]) in each environment, and also across environments (L-H-X), both

248 overall and for incross and outcross. Finally, adjacent environment pairs were contrasted

249 for among-cross $\mathrm{CV}$, low with high (L-H) and high with extreme $(\mathrm{H}-\mathrm{X})$, for each genetic

250 quality. The among-cross contrasts were conducted in the R-package 'cvequality' [52].

252 To explore the consistency of genetic lines across environments, another key aspect of G

$253 \mathrm{x}$ E interactions, genetic correlations $\left(r_{g}\right)$ across adjacent environments were calculated.

254 GLMMs were fitted with cross as a random effect and the variance component for cross

255 was extracted for each environment. GLMMs were then carried out between pairs of

256 adjacent environments ( $\mathrm{L}-\mathrm{H}, \mathrm{H}-\mathrm{X})$, with the cross $\mathrm{x} \mathrm{E}$ interaction included as a random

257 effect, and the interaction variance component extracted. As before, thorax length was

258 added as a fixed covariate to control for body size. An estimate of $r_{g}$ was then calculated

259 as:

260

261

$r_{g}=\sigma_{1,2} / \sqrt{ } \sigma^{2}{ }_{1,1} \sigma^{2}{ }_{2,2}$,

263 where $\sigma_{1,1}^{2}$ and $\sigma_{2,2}^{2}$ are the genetic variances in environments 1 and 2 respectively, and

$264 \sigma_{1,2}$ is the genetic covariance between the two environments [53]. Broad bounds of the $r_{\mathrm{g}}$

265 values were tested via model simplification and likelihood ratio tests (details in SI.A) 
(e) Statistical software used

268 All statistical analyses were conducted in JMP v.12.0.1 (SAS Institute 1989-2015) and R

269 v.3.4.2 [54]. GLMM tables, effect coefficients and extended methods are shown in SI.A.

\section{Results}

(a) Response in mean trait

274

As expected, male eyespan $\left(F_{2,45.47}=693.4, P<0.001\right)$ and thorax $\left(F_{2,41.80}=343.4 P<\right.$ 0.001) were smaller under higher environmental stress. The same was the case under genetic stress for eyespan $\left(F_{1,22.94}=4.783, P=0.028\right)$ but not for thorax, though its response was in the same direction $\left(F_{1,13.78}=3.222, P=0.095\right)$. After controlling for body

279 size variation, the same direction of change was observed in male eyespan for

280 environmental $\left(F_{2,54.66}=258.1, P<0.001\right)$ and genetic stress $\left(F_{1,7.421}=6.203, P=0.039\right)$.

281 All following comparisons report relative trait values.

282

283 In addition, there was a genetic quality-by-environment interaction $\left(F_{2,39.33}=5.379, P=\right.$

2840.009 , Figure 3a). The nature of the $\mathrm{G} \times \mathrm{E}$ was evident from comparison of adjacent environments. The difference in male eyespan between incross flies with low genetic quality and outcross flies with high genetic quality increased from low to high environmental stress (i.e. scale variance $\mathrm{G} \times \mathrm{E}, F_{1,18.35}=6.352, P=0.021$ ). But there was convergence between genetic quality classes after a further increase from high to extreme environmental stress (i.e. inverse scale variance $\mathrm{G} \times \mathrm{E}, F_{1,15.64}=8.664, P=0.010$ ). This pattern was confirmed by looking at environments separately. The difference between

291 incross and outcross male eyespan was evident at high $(t=8.65, \mathrm{df}=19.81, P<0.001)$, 
292 \# Figure 3.

293

294

295

296

297

298

299

300

301

302

303

304

305

306

307

308

309 Fig. 3. a) Male eyespan (least-squares mean $\pm \mathrm{SE})$ and $b)$ coefficient of variation (CV \pm

$31095 \% \mathrm{CI}$ ) across environmental stress (low, high and extreme) and genetic class, incross

311 (red) and outcross (blue). The red and blue lines are shown for illustrative purposes and

312 clarity. Asterisks denote significance: NS non-significant, ${ }^{*}<0.05,{ }^{* *} P<0.01, * * *<$

313 0.001. For CVs, the significance of incross versus outcross contrasts are displayed above

314 each food level category (black asterisk at the top). The significance of within incross

315 (red asterisks) and outcross (blue asterisks) contrasts are shown between pairs of adjacent

316 food levels. Incross and outcross lines are jittered for clarity. \# 
317 but absent at low $(t=1.98, \mathrm{df}=19.79, P=0.073)$ and extreme levels of environmental

318 stress $(t=-1.01, \mathrm{df}=21.87, P=0.298)$.

320 When comparisons were limited to incross lines, there were environmental $\left(\chi_{1}^{2}=276.7\right.$, $P<0.001)$ and genetic line differences $\left(\chi_{1}^{2}=11.08, P<0.001\right)$ but no $\mathrm{G} \times \mathrm{E}$ interaction $\left(\chi_{1}^{2}=4.281, P=0.509\right)$. A similar pattern was found in outcross lines, where there were environmental $\left(\chi_{1}^{2}=243.4, P<0.001\right)$ and genetic line differences $\left(\chi_{1}^{2}=5.14, P=0.023\right)$

324 but no $\mathrm{G} \times \mathrm{E}$ interaction $\left(\chi_{1}^{2}=7.71, P=0.173\right)$. These results indicate that $\mathrm{G} \times \mathrm{E}$ interactions were only apparent in the comparison of genetic quality (i.e. incross vs. outcross), and not in the comparison of genetic lines within low or high genetic quality groups.

\section{(b) Response in trait variance}

331 The genetic quality $\mathrm{G} \times \mathrm{E}$ pattern was further examined by looking at the among-cross variance in the response to stress. Coefficients of variation $(\mathrm{CV})$ were used to control for the positive scaling in variance due to changes in mean trait size. Male eyespan amongcross CV (Figure $3 b)$ was larger with greater environmental stress overall $\left(R_{M}=26.55, P\right.$

$335<0.001$ ), and separately for incross (incross $R_{M}=40.00, p<0.001$ ) and outcross lines

$336\left(R_{M}=130.35, P<0.001\right)$. But the extent of increase in $\mathrm{CV}$ from low to high

337 environmental stress was considerably more marked among incross males with low

338 genetic quality $\left(1.30 \%\right.$ increase, $\left.R_{M}=28.95, P<0.001\right)$ than outcross males with high

339 genetic quality $\left(0.23 \%\right.$ increase, $\left.R_{M}=11.95, P<0.001\right)$. Differences among outcross lines

340 were revealed to a much greater extent once the level of environmental stress increased

341 even further, in the transition from high to extreme environmental stress $(2.63 \%$ 
342 increase, $\left.R_{M}=57.34, P<0.001\right)$. This pattern contrasted again with males from incross

343 lines, where CV did not differ between high and extreme environmental stress levels

$344\left(0.78 \%\right.$ increase, $R_{M}=1.848, P=0.174$, Figure $\left.3 b\right)$. As for mean eyespan, the difference

345 between incross and outcross CV was seen only under high environmental stress (low

346 stress $R_{M}=0.814, P=0.367$, high stress $R_{M}=24.32, P<0.001$, extreme stress $R_{M}=$

$3471.148, P=0.284$; Figure 3b).

348

349 (c) Across environment genetic correlations

350

351 To further evaluate the role of male eyespan as a signal of genetic quality, we examined

352 whether genetic lines performing well in one environment performed well across all

353 environments (Figure 4), a critical part of the Gx E pattern. When pooling all lines,

354 there was a positive genetic correlation $\left(r_{\mathrm{g}}\right)$ between low and high $\left(r_{\mathrm{g}}=0.563, \chi_{1}^{2}=11.54\right.$, $P<0.001)$, and high and extreme environmental stress $\left(r_{\mathrm{g}}=0.360, \chi_{1}^{2}=22.94, P<\right.$

0.001). There was also a genetic correlation-by-environment interaction between low and high stress $\left(\chi_{1}^{2}=15.27, P<0.001\right)$ in which the genetic lines fanned out under higher environmental stress.

360 Analysing the two genetic quality classes separately, for low quality incross lines, genetic

361 correlations $\left(r_{\mathrm{g}}\right)$ were positive between low and high $\left(r_{g}=0.267, \chi_{1}^{2}=4.184, P=0.041\right)$,

362 as well as between high and extreme stress environments $\left(r_{g}=0.082, \chi_{1}^{2}=11.11, P<\right.$

363 0.001). For the high quality outcross lines, there was no genetic correlation between low

364 and high stress environments $\left(\chi_{1}^{2}=0.221, P=0.469\right)$, but $r_{\mathrm{g}}$ was positive between high

365 and extreme stress environments $\left(r_{g}=0.171, \chi_{1}^{2}=5.189, P=0.023\right)$. The lack of $r_{\mathrm{g}}$ was

366 due to severely reduced variation among outcross lines in the low $\left(\mathrm{CV}_{\text {low }}=0.188, \mathrm{CV}_{\text {high }}\right.$ 
a)

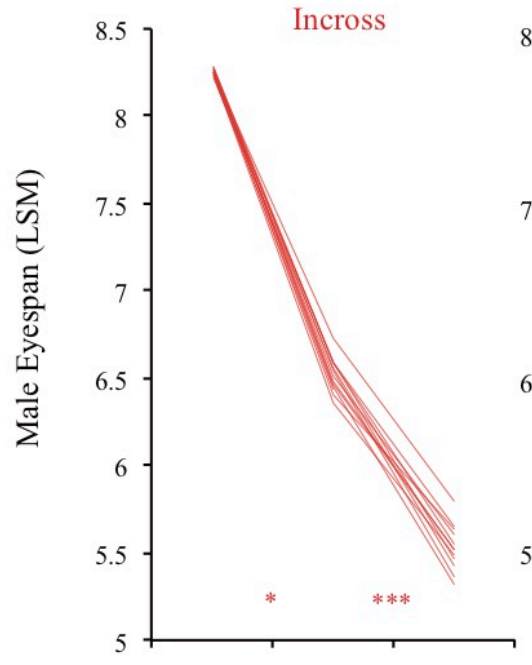

b)

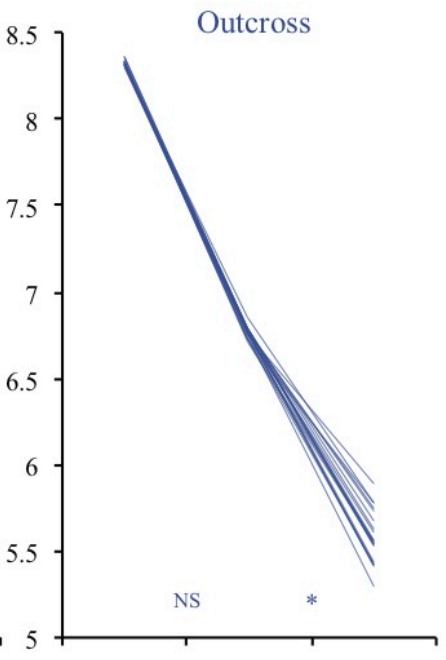

d) c)

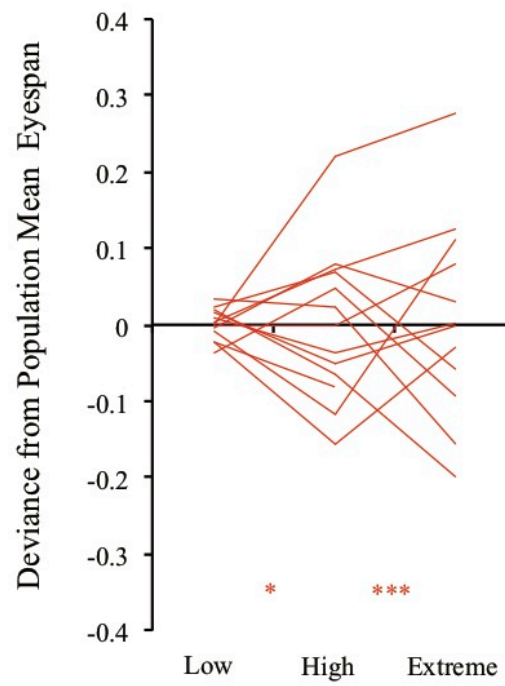

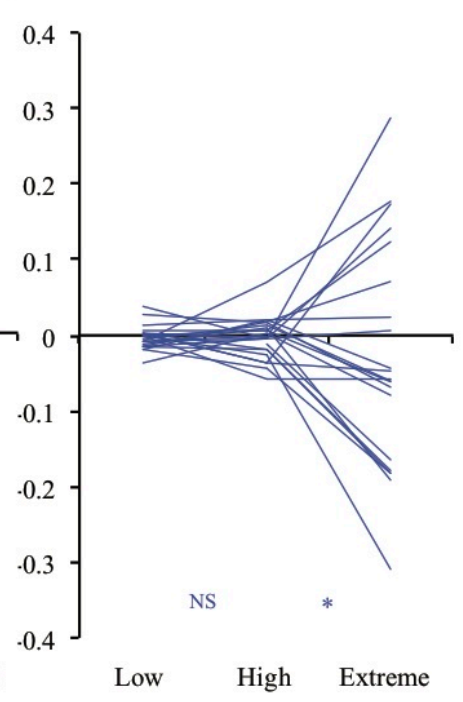

Food Stress Level

386 Fig. 4. Mean male eyespan (least-squares mean relative values) at each environmental

387 stress for each cross a) incross (red) and b) outcross lines (blue). Asterisks denote

388 significance of the effect of cross, NS non-significant, ${ }^{*} P<0.05, * * * P<0.001$.

389 An alternative representation is shown as the absolute deviation of each line from the c)

390 incross and d) outcross population mean. Error bars are excluded for clarity. \# 
$\left.392=0.416, \mathrm{CV}_{\text {extreme }}=3.05\right)$ compared to high $\left(R_{M}=57.34, P<0.001\right)$ or extreme stress

393 environments $\left(R_{M}=88.17, P<0.001 ;\right.$ Figure 4$)$.

394

395 (d) Survival across $\mathrm{G}$ and $\mathrm{E}$ stress

396 Larval survival was measured through a census of pupae. There was a survival effect of

$397 \mathrm{E}\left(F_{1,64.36}=64.36, P<0.001\right)$ but not of $\mathrm{G}\left(F_{1,20.04}=0.852, P=0.367\right)$ or $\mathrm{G} \times \mathrm{E}\left(F_{2,64.28}=\right.$

$3980.976, P=0.382$ ). The effect was a reduction in survival at extreme environmental stress

399 (pupae counts: $\mathrm{LSM} \pm \mathrm{SE}$ low $=2.17 \pm 0.10$, high $=2.29 \pm 0.09$, extreme $=1.55 \pm 0.09$ ).

400 A Tukey's HSD test confirmed that survival was lower under extreme relative to either

401 low or high environmental stress level $(P>0.05)$. Survival did not differ between incross

402 and outcross in any of these comparisons (all $P>0.05$, see SI.A).

403

404

405

\section{Discussion}

406

407 In this study we explicitly test whether environmental stress amplifies or obscures the

408 signal of genetic quality in male sexual ornaments. We do so in a unique way by direct

409 manipulation of both genetic quality and environmental stress, the latter over multiple

410 levels. The results enable us to put forward a unified explanation of how genetic and

411 environmental quality interact, advancing our understanding of the genetic benefits of

412 mate choice, with the potential to explain the diverse responses seen in other systems.

413

414 The response of male eyespan - the primary sexual ornament in D. meigenii - accords

415 with previous studies in stalk-eyed flies, showing that this male ornament is a sensitive

416 signal of both environmental $[9,11,35]$ and genetic stress $[1,24]$. Of greater interest, the 
417 new data captures a full range of $\mathrm{G} x \mathrm{E}$ interactions. The difference between low and

418 high genetic quality, in both eyespan mean and variance (coefficient of variation),

419 increases with the transition from low to high environmental food stress (Figure 3). This

420 is an example of "scale variance" $\mathrm{G} \times \mathrm{E}$ in which higher environmental stress amplifies

421 genetic differences. It has been observed across a range of species, for example in

422 structural wing pigmentation (UV angular visibility) in the butterfly Colias eurytheme [55],

423 male song attractiveness in the lesser waxmoth, Achroia grisella [2], and attractiveness

424 traits in the black scavenger fly, Sepsis punctum [15], all examples of traits associated with

425 sexual success. In contrast, the difference between our low and high genetic quality

426 classes, in both eyespan mean and variance, decreases with the transition from high to

427 extreme environmental food stress (Figure 3). This reversed pattern is an example of

428 "inverse scale variance" $\mathrm{G}$ x $\mathrm{E}$ in which stress denudes genetic differences. It again has

429 been observed across a range of species, for example, iridescent and orange area in the

430 guppy Poecilia reticulata [17], cuticular hydrocarbon blend in Drosophila simulans [16], and

431 to a more limited extent, UV brightness in C. eurytheme [55].

433 Our results are novel and striking because we see both scale variance and inverse scale

434 variance in the same trait in a single species. This leads us to propose a unified

435 hypothesis for G x E interactions in signals of quality. Moderate to large increases in

436 environmental stress lead to amplification of the phenotypic expression of genetic

437 quality, whereas as environmental stress becomes extreme, increases in phenotypic

438 variation overwhelm the underlying genetic differences in quality. We note that in some

439 previous studies, separate traits respond differently to environmental stress, suggesting

440 variation in the threshold at which amplification transitions to restriction (e.g. $[2,55])$. 
441 Future studies will be needed to identify which characteristics are associated with

442 sensitivity levels in different traits, and whether these relate to costs of trait expression.

443

444 Yet, some evidence from other studies of sexual ornaments seems to contradict the

445 unified hypothesis which report no interaction between genetic and environmental stress,

446 for example in morphological traits and cuticular hydrocarbons in D. melanogaster [40]

447 and several sexual traits in $P$. reticulata guppies [39]. Both of these experiments examined

448 groups that differ predictably in genetic quality (hemiclonal lines and inbred versus

449 outbred lines, respectively). But the lack of response likely reflects the application of

450 insufficiently intrusive environmental stress. For example, the "stressful" environment in

451 guppies was a moderate density [39], while that in D. melanogaster was a minor reduction

452 to $70 \%$ of the normal diet [40]. A previous study in stalk-eyed flies likewise found little

453 impact of food reduction of this order [11]. For comparison, our dilution for extreme

454 stress was a restriction to just $5 \%$ of the standard diet. Moreover, as each of these studies

455 used just two levels of environmental stress, analysis of complex G x E was precluded.

456 This is not a criticism of either study, which had different goals to ours, but highlights

457 that neither would provide an adequate test of our hypothesis. Another commonly

458 reported pattern across diverse species, also potentially at odds with our interpretation, is

459 "crossover" G x E in which different genetic lines are superior in different environments.

460 This has been shown for male signal rate in the lesser waxmoth [19] and song traits of

461 Enchenopia treehoppers [20]. However, "crossover" G x E is not really a distinct

462 category, and can co-occur with "scale" or "inverse scale" G x E patterns [56]. For

463 instance, crossover embedded within $\mathrm{G} \times \mathrm{E}$ scale variance patterns in the lesser

464 waxmoth [2] and inverse scale variance in the guppy [17] has been observed. Once

465 again, the interpretation that crossover dominates the $\mathrm{G} \times \mathrm{E}$ pattern requires 
investigation of sufficient levels of environmental stress relative to the traits in question.

467 Without this, crossover should only be seen as part of $\mathrm{G} \times \mathrm{E}$ response, exerting ambiguous limits on the signalling function of the sexual ornament.

470 It is vitality important to examine a range of environments from low through to an

471 extreme form of stress, alongside similar dimensions of genetic quality variation. Distinct

472 classes of environmental quality variation were created in a standard manner through

473 food restriction applied to developing larvae $[1,11,15,30,40,45]$. These treatments

474 differed from previous studies in the use of food dilution to an "extreme", defined as the

475 point before larval survival showed a clear-cut decline (Figure 2). The reason for

476 choosing this point was in part logistical, in order to easily collect similar sample size

477 across the different stress levels. We also wanted to avoid the possibility that differential

478 survival causes changes in trait mean and variation across the different genetic quality

479 and environmental stresses. Despite this precaution, there was a moderate effect of the

480 extreme environmental stress on larval survival. This could have contributed to the trait

481 patterns observed if there was a lower level cut-off in the eyespan of survivors. We

482 suspect this effect was minor as the mean was lowest and the CV highest in the extreme

483 environment (Fig. 3), and more importantly, the survival deficit was equal across incross

484 and outcross flies. Our conclusions appear to be robust. Our use of food quantity as an

485 environmental stress was for its ease of manipulation and its use in many previous

486 studies. Competition for food is likely to be a factor in many species and so we suspect

487 that the results we report here are general stress responses. This needs to be established

488 through comparison with other stresses, such as fluctuations in temperature, $\mathrm{pH}$ or food

489 quality, that are part of the normal range of environmental stress in the wild [57]. 
491 To create distinct classes of genetic quality, a set of highly homozygous inbred lines

492 (incross) were compared against crosses between lines (outcross) which are predicted to

493 be highly heterozygous for the mutational load carried by incross lines. In the pilot

494 experiment (Fig. 2), as well as the actual experiment, there was no difference in egg-to-

495 adult survival between flies in the incross and outcross genetic quality treatments. The

496 lack of a viability difference suggests that there was a strong purging of deleterious alleles

497 during the creation of the inbred lines, as is expected and observed in other studies [58-

498 60]. Our objective was not to study the inbreeding per se, as this is unlikely to be the

499 object of female mate preference in this species. Rather we use inbreeding status as an

500 investigative tool, in order to uncover the full nature of genetic quality-by-environment

501 interactions on variation in signal trait size. In particular, previous $\mathrm{G}$ x $\mathrm{E}$ studies have

502 failed to use a sufficient range of variation in genetic quality. A typical approach is to use

503 distinct genetic lines, like brother-sister families [1,20] or inbred lines [2]. But groups that

504 differ predictably in genetic quality have not been examined properly against a wide

505 range of environmental stress [39-40]. Independent lines provide information about

506 genetic variation but may differ only slightly, and unpredictably, in genetic quality, and

507 then only with differences established post hoc. In our study, we distinguish between

508 variation in genetic quality in the comparison of incross and outcross flies, and genetic

509 variation between lines within these quality categories. In accordance with prior studies

510 [1], our results show differences in performance between lines. Crucially, there was no

511 among-line $\mathrm{G} \times \mathrm{E}$ once analysis was limited to a particular genetic quality class, both for

512 incross and outcross. The set of lines in each genetic quality class appear to have been

513 sufficiently similar in quality that they responded in an equivalent manner when

514 challenged with our wide range of environmental stress levels (Figure 4). Only the

515 comparison between incross and outcross flies revealed a strong $\mathrm{G} \times \mathrm{E}$ interaction, in 
516 which high quality (outcross) line resisted the effect of high but not extreme

517 environmental stress.

518

519 Taking the results together allows us to comment on sexual selection on males and the

520 potential indirect genetic benefits that arise from female mate choice. We expect sexual

521 selection to be severely attenuated under benign and extreme environmental stress, but

522 strong in high stress environments which amplify genetic quality differences. As stress is

523 likely to be the norm under common ecological conditions in nature, sexual selection

524 could often be stronger than currently estimated from laboratory experiments - typically

525 carried out under low stress conditions of ad libitum food, constant temperature, no

526 predators and parasites, and no ecological competitors. We note that our

527 experimentation used stress from a unimodal environment variable (food availability),

528 controlling all other physical and biotic factors, and that we used a simple measure of

529 male signalling, leaving aside other, more subtle aspects of male behaviour used in

530 female evaluation of their partners [46]. This implies that benign environmental

531 conditions, equivalent to low stress in our experiment (i.e. in which larvae have excess

532 food and little competition), are rare. Extreme environments are likewise also likely to be

533 rare as they are not those that maintain viable populations. The majority of

534 environments probably lie between the low and high regimes, which is consistent with

535 the considerable range in eyespan observed among wild caught stalk-eyed flies [32].

536

537 The outcome in nature for female choice will depend on the distribution of

538 environmental stress, its spatial and temporal variability, and hence its consequence for

539 the pool of available mates in a given population [12]. If conditions can be categorised as

540 low, high or extreme, then the indirect benefits of mate choice will be greatest in high 
541 stress environments, as these bring out genetic differences to the greatest extent. As

542 genetic line correlations across environments were positive (with the exception of

543 outcross lines between low and high food stress, where a lack of variation precluded

544 reliable calculation), genetic differences will be evident to some extent in all

545 environments. Where environmental conditions in a population are a mixture of low,

546 high and extreme, individuals with the most exaggerated sexual ornaments will be an

547 assortment of those with high genetic quality from a range of environments diluted by

548 those less well genetically endowed but who experienced lower environmental stress

549 during development. This cuts at the indirect genetic benefits but nonetheless there will

550 be advantage to female mate choice. To conclude, while environmental variation places

551 contingencies on signalling, sometimes amplifying and sometimes muting its value,

552 genetic variation in quality between individuals will always to some extent be evident in

553 the sexual ornament and feed through to their offspring. 
556 Data accessibility. Data are made available at the Dryad Digital Repository [TO ADD].

558 Author contributions. JMH, KF and AP conceptualised the study and methodology,

559 and wrote, reviewed and edited the paper. The formal analysis was carried out by $\mathrm{JMH}$,

560 who with HACD carried out the experiments. Stalk-eyed fly resources were provided by

$561 \mathrm{AP}$ and $\mathrm{KF}$, who secured funding and supervised the project.

562

563 Competing interests. The authors declare no competing interests.

564

565 Acknowledgements. The authors acknowledge support for JMH by a NERC

566 Studentship, AP by EPSRC grants (EP/F500351/1, EP/I017909/1), and AP and KF by

567 a NERC grant (NE/G00563X/1, NE/R010579/1). We thank Hans Feijen for sharing

568 data from natural populations of Diasemopsis meigenii. Additional experimental support

569 was provided by Rebecca Finlay, Koichi Yamanoha, and Anna Aichinger who also

570 assisted in figure production. We acknowledge the work in creating and maintaining the

571 inbred lines used in this work by Lawrence Bellamy, Nadine Chapman, David Ellis and

$572 \quad$ Luke Lazarou.

573

574

575 SUPPLEMENTAL INFORMATION

576 Supplemental information includes all details of statistical effect size estimates for the

577 tests of mean effects, and additional method details. [Available after formal publication.] 


\section{References}

1. David P, Bjorksten T, Fowler K, Pomiankowski A. 2000 Condition-dependent signalling of genetic variation in stalk-eyed flies. Nature 406, 186-188 (doi: $\underline{10.1038 / 35018079)}$.

2. Danielson-François AM, Kelly JK, Greenfield MD. 2006 Genotype x environment interaction for male attractiveness in an acoustic moth: evidence for plasticity and canalization. J. Evol. Biol. 19, 532-542 (doi:10.1111/j.1420-9101.2005.01006.x).

3. Pomiankowski A. 1988 The evolution offemale mating preferences for male genetic quality. 136-184 (New York: Oxford University Press).

4. Houle D. 1992 Comparing evolvability and variability of quantitative traits. Genetics 130, 195-204 (pmid:32160).

5. Pomiankowski A, Møller, AP. 1995 A resolution of the lek paradox. Proc. R. Soc. Lond. B 260, 21-29 (doi:10.1098/rspb.1995.0054).

6. Jennions MD, Petrie M. 2000 Why do females mate multiply? A review of the genetic benefits. Biol. Rev. Camb. Philos. Soc. 75, 21-64 (doi:10.1111/j.1469185X.1999.tb00040.x).

7. Taylor ML, Wedell N, Hosken DJ. 2007 The heritability of attractiveness. Curr. Biol. 17, R959-R960 (doi:10.1016/j.cub.2007.09.054).

8. Zuk M, Thornhill R, Ligon, JD. 1990 Parasites and mate choice in red jungle fowl. Am. Zool. 30, 235-244 (pmid:10319255).

601 
604 10. Kotiaho JS. 2000 Testing the assumptions of conditional handicap theory: costs and condition dependence of a sexually selected trait. Behav. Ecol. Sociobiol. 48, 188194. (doi:10.1007/s002650000221).

11. Cotton S, Fowler K, Pomiankowski A. 2004a Condition dependence of sexual ornament size and variation in the stalk-eyed fly Cyrtodiopsis dalmanni (Diptera : Diopsidae). Evolution 58, 1038-1046 (doi:10.1111/j.0014-3820.2004.tb00437.x).

12. Greenfield MD, Rodriguez RL. 2004 Genotype-environment interaction and the reliability of mating signals. Anim. Behav. 68, 1461-1468 (doi:10.1016/j.anbehav.2004.01.014).

13. Bussière LF, Hunt J, Stölting KN, Jennions M, Brooks R. 2008 Mate choice for genetic quality when environments vary: suggestions for empirical progress. Genetica 134, 69-78 (doi:10.1007/s10709-007-9220-z). (doi:10.1007/s10709-008-9297-z).

14. Danielson-François AM, Zhou Y, Greenfield MD. 2009 Indirect genetic effects and the lek paradox: inter-genotypic competition may strengthen genotype $\mathrm{x}$

16. Ingleby FC, Hunt J, Kosken DJ. 2013 Genotype-by-environment interactions for cuticular hydrocarbon expression in Drosophila simulans. J. Evol. Biol. 26, 94-107 (doi:10.1371/journal.pone.0067623). 
629 18. Charmantier, A. \& Garant, D. 2005. Environmental quality and evolutionary potential: lessons from wild populations. Proc. $R$. Soc. B, 272, 1415-1425 (doi:10.1098/rspb.2005.3117).

19. Jia FY, Greenfield MD, Collins RD. 2000 Genetic variance of sexually selected traits in waxmoths: maintenance by genotype x environment interaction. Evolution 54, 953-967 (doi:10.1111/j.0014-3820.2000.tb00095.x).

20. Rodríguez RL, Al-Wathiqui N. 2011 Genotype x environment interaction is weaker in genitalia than in mating signals and body traits in Enchenopa treehoppers (Hemiptera: Memracidae). Genetica 139, 871-884 (doi:10.1007/s10709-011-9591-

21. Etges WJ, de Oliveira CC, Gragg E, Ortíz-Barrientos D, Noor MA, Ritchie MG. 2007. Genetics of incipient speciation in Drosophila mojaveneis. I. Male courtship

22. Hoffman AA, Merilä J. 1999 Heritable variation and evolution under favourable and unfavourable conditions. TREE 14, 96-101 (doi:10.1016/S0169-5347(99)015955).

646 23. Cotton S, Rogers DW, Small J, Pomiankowski A, Fowler K. 2006 Variation in preference for a male ornament is positively associated with female eyespan in the stalk-eyed fly Diasemopsis meigenii. Proc. R. Soc. Lond. B 273, 1287-1292

650 24. Bellamy L, Chapman N, Fowler K, Pomiankowski A. 2013 Sexual traits are sensitive to genetic stress and predict extinction risk in the stalk-eyed fly, 
653 25. Burkhardt D, de la Motte I. 1988 Big 'antlers' are favoured: female choice in stalkeyed flies (Diptera, Insecta), field collected harems and laboratory experiments. $J$. Comp. Physiol. A 162, 649-652 (doi:10.1007/BF01342640).

26. Burkhardt D, de la Motte, I, Lunau K. 1994 Signalling fitness: larger males sire more offspring. Studies of the stalk-eyed fly Cyrtodiopsis whitei (Diopsidae, Diptera). J. Comp. Physiol. A 174, 61-64 (doi:10.1007/BF00192006).

27. Wilkinson GS, Reillo PR. 1994 Female choice response to artificial selection on an exaggerated male trait in a stalk-eyed fly. Proc. R. Soc. Lond. B 255, 1-6 (doi:10.1098/rspb.1994.0001).

28. Wilkinson GS, Dodson GN. 1997 Function and evolution of antlers and eye stalks in flies. In: The evolution of mating systems in insects and arachnids (eds. Choe, J \& Crespi, B). 310-328 (Cambridge: Cambridge University Press).

29. Wilkinson GS, Kahler H, Baker RH. 1998 Evolution of female mating preferences in stalk-eyed flies. Behav. Ecol. 9, 525-533 (doi:10.1093/beheco/9.5.525).

30. Hingle A, Fowler K, Pomiankowski A. 2001a The effect of transient food stress on female mate preference in the stalk-eyed fly Cyrtodiopsis dalmanni. Proc. R. Soc. Lond. B 268, 1239-1244 (doi:10.1098/rspb.2001.1647).

31. Hingle A, Fowler K, Pomiankowski A. 2001b Size-dependent mate preference in the stalk-eyed fly Cyrtodiopsis dalmanni. Animal Behav. 61, 589-595 (doi:10.1006/anbe.2000.1613).

32. Cotton S, Small J, Hashim R, Pomiankowski A. 2010 Eyespan reflects reproductive quality in wild stalk-eyed flies. Evol. Ecol. 24, 83-95 (doi:10.1007/s10682-0099292-6). 
678 34. Panhuis TM, Wilkinson GS. 1999 Exaggerated male eye span influences contest outcome in stalk-eyed flies (Diopsidae). Behav. Ecol. and Sociobiol. 46, 221-227 (doi:10.1007/s002650050613).

681

35. Cotton S, Fowler K, Pomiankowski A. 2004b Heightened condition dependence is not a general feature of male eyespan in stalk-eyed flies (Diptera : Diopsidae). $J$. Evol. Biol. 17, 1310-1316 (doi:10.1111/j.1420-9101.2004.00754.x).

36. Prokop ZM, Les JE, Banas PK, Koteja P, Radwan J. 2010 Low inbreeding depression in a sexual trait in the stalk-eyed fly Teleopsis dalmanni. Evol. Ecol. 24, 827-837 (doi:10.1007/s10682-009-9341-1).

37. Knell RJ, Fruhauf N, Norris K.A. 1999 Conditional expression of a sexually selected trait in the stalk-eyed fly Diasemopsis aethiopica. Ecol. Entomol. 24, 323-328 (doi:10.1046/j.1365-2311.1999.00200.x).

38. Bjorksten TA, Pomiankowski A, Fowler K. 2011 Temperature shock during development fails to increase the fluctuating asymmetry of a sexual trait in stalkeyed flies. Proc. R. Soc. Lond. B. 268, 1503-1510 (doi:10.1098/rspb.2001.1575).

39. Zajitschek SRK, Brooks RC. 2010 Inbreeding depression in male traits and preference for outbred males in Poecilia reticulata. Behav. Ecol. 21, 884-891 (doi:10.1093/beheco/arq077).

40. Bonduriansky R, Mallet MA, Arbuthnott D, Pawlowsky-Glahn V, Egozcue JJ, Rundle HD. 2015 Differential effects of genetic vs. environmental quality in Drosophila melanogaster suggest multiple forms of condition dependence. Ecol. Lett. 18, 317-326 (doi:10.1111/ele.12412).

41. Bellamy L, Fowler K, Pomiankowski A. 2014 The use of inbreeding to assess the genetic component of condition underlying GEIs in sexual traits. In Genotype-by-Environment 
42. Falconer DS, Mackay TFC. 1996 Introduction to quantitative genetics (New York: Longman Scientific \& Technical).

43. Kolss M, Vijendravarma RK, Schwaller G, Kawecki TJ. 2009 Life history 2389-2401 (doi:10.1111/j.1558-5646.2009.00718.x).

44. Sisodia S, Singh BN. 2012 Experimental evidence for nutrition regulated stress resistance in Drosophila ananassae. PLoS One 7, e46131 (doi:10.1371/journal.pone.0046131).

45. Burger JMS, Hwangbo DS, Corby-Harris V, Promislow DE. 2007 The functional costs and benefits of dietary restriction in Drosophila. Aging Cell 6, 63-71 (doi:10.1111/j.1474-9726.2006.00261.x).

46. Chapman NC, Siriwat P, Howie J, Towlson A, Bellamy L, Fowler K, Pomiankowski A. 2017. The complexity of mating decisions in stalk-eyed flies. sexual ornament size is positively associated with reproductive morphology and enhanced fertility in the stalk-eyed fly Teleopsis dalmanni. BMC Evol. Biol. 8, 236 (doi:10.1186/1471-2148-8-236).

48. Meade L, Harley E, Howie JM, Pomiankowski A, Fowler K. 2017 Variation in the benefits of multiple mating on female fertility in wild stalk-eyed flies. Ecol. Evol. 7, 10103-10115 (doi:10.1002/ece3.3486). image analysis. Nat. Methods 9, 671-675 (pmid:22930834). 
50. Rowínski PK, Rogell B. 2017 Environmental stress correlates with increases in both genetic and residual variances: a meta-analysis of animal studies. Evolution 71, 1339-1351 (doi:10.1111/evo.13201).

51. Krishnamoorthy K, Lee M. 2014 Improved tests for the equality of normal coefficients of variation. Comput. Stat. 29, 215-232 (doi:10.1007/s00180-0130445-2).

52. Marwick B, Krishnamoorthy K. 2016 Tests for the equality of coefficients of variation from multiple groups. R package, version 0.1.1. http://CRAN.Rproject.org $/$ package $=$ quantreg

53. Roff DA, Wilson AJ. 2014 Quantifying genotype-by-environment interactions in laboratory systems. In Genotype-by-Environment Interactions and Sexual Selection, J. (eds. Hunt \&

54. R Core Development Team. 2017 R: A language and environment for statistical computing. R Foundation for Statistical Computing, Vienna, Austria.

55. Kemp DJ, Rutowski RL. 2007 Condition dependence, quantitative genetics, and the potential signal content of iridescent ultraviolet butterfly coloration. Evolution $\mathbf{6 1}$, 168-183 (doi:10.1111/j.1558-5646.2007.00014.x ).

56. Ingleby FC, Hunt J, Hosken DJ. 2010 The role of genotype-by-environment

747 57. Hoffmann AA, Parsons PA. 1993 Evolutionary Genetics and Environmental Stress (Oxford: Oxford University Press).

749 58. Crow JF, Kimura M. 1970 An Introduction to Population Genetic Theory (New York: Harper and Row). 
751 59. Crnokrak P, Barrett SCH. 2002 Purging the genetic load: a review of the experimental evidence. Evolution 56, 2347-2358 (doi: 10.1534/genetics.111.135541).

754 60. García-Dorado A. 2012. Understanding and predicting the fitness decline of shrunk 